\title{
Comparison of Elastic Intramedullary Nails and Locking Compression Plates on Oxidative Stress in Children with Distal Tibial Metaphyseal Fractures
}

\author{
Yupeng $\mathrm{Xu}$, Guoyuan Huang and Cunliang Niu \\ Second Department of Orthopedics, Wuwei People's Hospital, Gansu, 733000, China
}

\begin{abstract}
The aim of this study was to compare the effects of elastic intramedullary nails and locking compression plates on oxidative stress like superoxide dismutase (SOD), total antioxidant capacity (TAC), catalase (CAT), and malondialdehyde (MDA) in children with distal tibial metaphyseal fractures. It was an experimental study carried out from January 2012 to October 2017. Ninety-eight children with distal tibial metaphyseal fractures were randomly divided into group A and group B, 49 cases in each group. Group A was treated with elastic intramedullary nails and group B with locking compression plates. The research showed that operation time of group A was shorter than those of group $B(p<0.001)$, incision complication rate of group A was lower than that of group $B(p=0.025)$, and the American Orthopaedic Foot and Ankle Society (AOFAS) ankle-hindfoot score of group A was higher than that of group B $(p<0.001)$ after 12 months of follow-up. On the $7^{\text {th }}$ day after operation, the levels of SOD, TAC and CAT in group A were higher than those in group $B(p=0.003$, $p<0.001, p<0.001$, respectively), but level of serum MDA in group $A$ was lower than that in group $B(p<0.001)$. Compared with locking compression plate, elastic intramedullary nail has better short-term effect, less operative time, lower incision complications, and less influence on oxidative stress.
\end{abstract}

Key Words: Children, Distal tibial fracture, Metaphysis, Intramedullary nail, Locking plate, Oxidative stress.

How to cite this article: Xu Y, Huang G, Niu C. Comparison of elastic intramedullary nails and locking compression plates on oxidative stress in children with distal tibial metaphyseal fractures. J Coll Physicians Surg Pak 2019; 29(11):1118-20.

The treatment of distal tibial metaphyseal fracture is an important challenge. 1 Conservative treatment of distal tibial metaphyseal fracture in children is not effective due to the influence of many factors, such as the inadaptability of children to long-term traction and plaster fixation, the relatively poor ability of fracture shaping, and the less coverage of subcutaneous soft tissue in the lower tibial segment of children. In theory, both elastic intramedullary nails and locking compression plates are important treatments for distal tibial metaphyseal fractures. As the fracture approaches or extends to the ankle, the stability of intramedullary nail is reduced; while as the tibia lacks muscle coverage, the use of locking compression plate is prone to occurring incision problems. Therefore, the optimal treatment of distal tibial metaphyseal fractures in children remains controversial. ${ }^{2}$ Surgery can cause intense oxidative stress in the body. Observing the changes of oxidative stress can understand the degree of injury to the body caused by operation. Research has suggested that oxidative stress occurs after a fracture and it may be proportional to the number of bones fractured. ${ }^{3}$ Superoxide dismutase

Correspondence to: Cunliang Niu, Second Department of Orthopedics, Wuwei People's Hospital, Gansu, 733000, China E-mail:kmqd16@163.com

Received: December 4, 2018; Revised; February 26, 2019

Accepted: April 08, 2019
(SOD), total antioxidant capacity (TAC), catalase (CAT) and malondialdehyde (MDA) are common indicators of oxidative stress.

At present, there are few reports comparing the effects of elastic intramedullary nails and locking compression plates on oxidative stress in children with distal tibial metaphyseal fractures.

The aim of this study was to compare the effects of elastic intramedullary nails and locking compression plates on oxidative stress in children with distal tibial metaphyseal fractures. This experimental study was carried out at the Second Department of Orthopedics, Wuwei People's Hospital, China, from January 2012 to October 2017. This study was approved by the Hospital Ethics Committee. Ninety-eight children with distal tibial metaphyseal fractures admitted to our hospital were selected as the research subjects. The criteria for inclusion were children confirmed as distal tibial metaphyseal fractures, closed or Gustilo type I fractures, and all operations were performed within 48 hours after injury. The exclusion criteria were children younger than 7 years and older than 16 years, open fractures of Gustilo type II and III, fracture line less than $1.0 \mathrm{~cm}$ from distal tibial epiphyseal plate, combined with nerve and blood vessel injuries, combined with other types of fractures like fibular fractures and so on, and children with surgical contraindications. Ninty-eight cases were randomly 
Table I: Comparison of the surgical results between two groups.

\begin{tabular}{|c|c|c|c|}
\hline Parameters & Group A $(n=49)$ & Group B $(n=49)$ & p-value \\
\hline Operation time (min) & $103.35 \pm 7.86$ & $126.41 \pm 9.75$ & $<0.001$ \\
\hline SOD before operation ( $\mathrm{nU} / \mathrm{mL})$ & $87.26 \pm 6.29$ & $87.33 \pm 7.12$ & 0.959 \\
\hline SOD at 7 days after operation $(\mathrm{nU} / \mathrm{mL})$ & $76.14 \pm 5.23$ & $72.57 \pm 6.18$ & 0.003 \\
\hline TAC before operation (kU/L) & $13.78 \pm 1.61$ & $13.82 \pm 1.75$ & 0.907 \\
\hline TAC at 7 days after operation (kU/L) & $10.39 \pm 1.02$ & $8.56 \pm 0.97$ & $<0.001$ \\
\hline CAT before operation ( $\mathrm{nU} / \mathrm{mL}$ ) & $45.14 \pm 4.38$ & $45.53 \pm 4.62$ & 0.669 \\
\hline CAT at 7 days after operation $(\mathrm{nU} / \mathrm{mL})$ & $37.65 \pm 3.29$ & $34.81 \pm 3.94$ & $<0.001$ \\
\hline MDA before operation ( $\mu \mathrm{mol} / \mathrm{L})$ & $2.25 \pm 0.22$ & $2.23 \pm 0.18$ & 0.623 \\
\hline MDA at 7 days after operation $(\mu \mathrm{mol} / \mathrm{L})$ & $3.86 \pm 0.78$ & $4.94 \pm 0.63$ & $<0.001$ \\
\hline Incidence rate of incision complications [n(\%)] & $2(4.08)$ & $9(18.37)$ & 0.025 \\
\hline AOFAS score after 12 months of follow-up (score) & $88.81 \pm 3.94$ & $82.47 \pm 3.36$ & $<0.001$ \\
\hline
\end{tabular}

divided into group A and group B with 49 cases in each group.

Group A was treated with elastic intramedullary nails, i.e. under general anesthesia, the incision was made at $1.5-2.0 \mathrm{~cm}$ of the proximal epiphyseal plate of the tibia with manual sharp cone. The pre-curved elastic intramedullary nail was placed into the incision, and the intramedullary position of the intramedullary nail was determined under the fluoroscopy of "C" arm machine, and manual reduction was performed. Double elastic intramedullary nails were used. The elastic intramedullary nail was placed at the distal end of the fracture and the proximal epiphyseal plate of the talus and tibia was about $0.5 \mathrm{~cm}$. The same diameter elastic intramedullary nail was then placed on the opposite side. When the fluoroscopy reduction of " $\mathrm{C}$ " arm machine was good, the intramedullary nail was cut and the entrance was exposed about $3.0 \mathrm{~cm}$. The force line of lower limbs was measured after operation to ensure that there was no rotation deformity in the calves.

Group B was treated with locking compression plate. Tibial plating was done by minimally invasive percutaneous plate osteosynthesis technology; namely, indirect reduction of distal tibial metaphyseal fracture by traction under general anesthesia. The steel plate was placed in medial tibia through subcutaneous tunnel and closed reduction was performed under $\mathrm{C}$-arm fluoroscopy. The plate was fixed internally and the incision was closed when it is satisfied. Both groups of operations were performed by the same operating surgeon.

The operation time was compared between the two groups. Seven days before and after the operation, $3 \mathrm{~mL}$ of peripheral venous blood was collected and serum was centrifuged. The changes of SOD, TAC, CAT and MDA were detected and compared by enzyme-linked immunosorbent assay (ELISA). The incidence of incision complications was observed and recorded. After 12 months of follow-up, the American Orthopaedic Foot and Ankle Society (AOFAS) ankle-hindfoot score of the two groups were compared to evaluate the ankle function of the children. Collected data were analysed using independent sample t-test and Chi-square test by Statistical
Package for Social Sciences (SPSS) version 25. P-value of less than 0.05 showed significant difference.

Among 98 children, 55 were males $(56.12 \%)$ and 43 were females $(43.88 \%)$, the age was ranged from 7 to 15 years with an average age of $12.54 \pm 2.65$ years. The causes of injury were: 34 cases of traffic injuries $(34.69 \%), 51$ cases of fall injuries $(52.04 \%)$, and 13 cases of high falling injuries $(13.27 \%)$. There were 9 cases $(9.18 \%)$ of transverse fracture, 40 cases $(40.82 \%)$ of oblique fracture, 30 cases $(30.61 \%)$ of spiral fracture, and 19 cases (19.39\%) of comminuted fracture. According to $\mathrm{AO}$ classification criteria of distal tibial fractures, A1 type was 46 cases (46.94\%), A2 type was 43 cases $(43.88 \%)$, and $A 3$ type was 9 cases $(9.18 \%)$.

Operation time of group $A$ was shorter than those of group B $(p<0.001)$, incidence rate of incision complications of group $A$ was lower than that of group $B$ $(p=0.025)$, and AOFAS score of group A was higher than that of group B ( $p<0.001$, Table I) after 12 months of follow-up.

Before operation, there was no significant difference in serum oxidative stress indicators between the two groups $(p=0.959,0.907,0.669,0.623)$. On the $7^{\text {th }}$ day after operation, the levels of SOD, TAC and CAT in serum of the two groups decreased slightly, but the levels of SOD, TAC and CAT in group $A$ were higher than those in group $B$ $(p=0.003, p<0.001, p<0.001)$. The level of serum MDA in two groups was slightly higher than that before operation, but the level of serum MDA in group A was lower than that in group $B(p<0.001$, Table $\mathrm{I})$.

This study showed that the operation time of elastic intramedullary nail was shorter than that of locking compression plate, which was consistent with previous studies.4 Dogra et al. considered that elastic intramedullary nails were safe and reliable in the treatment of distal tibial metaphyseal fractures. 5 Guo et al. pointed out that both elastic intramedullary nails and locking compression plates could be used safely to treat distal tibial metaphyseal fractures, but the former had the advantages of short operation time and easy removal of implants. ${ }^{6}$ This study found that elastic intramedullary nail was superior to locking compression plate in terms of 
incision complication rate and ankle function recovery after operation. The author found that elastic intramedullary nails had less effect on oxidative stress compared with locking compression plates. The reason may be that compared with elastic intramedullary nails, the treatment of locking compression plates has a bigger trauma, and is easier to activate neutrophils in vivo, produce more oxygen-free radicals, make lipid peroxidation, and then induce increased consumption of SOD, TAC, CAT, and increase production of MDA.

To sum up, compared with locking compression plate, elastic intramedullary nail has better short-term effect, less operative time, lower incision complications, and less influence on oxidative stress in the treatment of children with distal tibial metaphyseal fractures.

\section{CONFLICT OF INTEREST:}

Authors declared no conflict of interest.

AUTHORS' CONTRIBUTION:

YX: Devoted to collecting and interpreting the data, drafted the mansucript, responsible for the conception and design of the study.

$\mathrm{GH}$ : Devoted to collecting and interpreting the data.
$\mathrm{CN}$ : Devoted to collecting and interpreting the data, revised it critically for important intellectual content.

\section{REFERENCES}

1. Lqbal HJ, Pidikiti P. Treatment of distal tibia metaphyseal fractures; plating versus intramedullary nailing: A systematic review of recent evidence. Foot Ankle Surg 2013; 19:143-7.

2. Mahmood A, Kumar G. Review of the treatment of distal tibia metaphyseal fractures; plating versus intramedullary nailing: A systematic review of recent evidence. Foot Ankle Surg 2014; 20:151.

3. Prasad G, Dhillon MS, Khullar M, Nagi ON. Evaluation of oxidative stress after fractures. A preliminary study. Acta Orthop Belg 2003; 69:546-51.

4. Im GI, Tae SK. Distal metaphyseal fractures of tibia: A prospective randomized trial of closed reduction and intramedullary nail versus open reduction and plate and screws fixation. J Trauma 2005; 59:1219-23.

5. Dogra AS, Ruiz AL, Thompson NS, Nolan PC. Dia-metaphyseal distal tibial fractures - treatment with a shortened intramedullary nail: A review of 15 cases. Injury 2000; 31:799-804.

6. Guo JJ, Tang N, Yang HL, Tang TS. A prospective, randomised trial comparing closed intramedullary nailing with percutaneous plating in the treatment of distal metaphyseal fractures of the tibia. J Bone Joint Surg Br 2010; 92:984-8. 